\title{
Saúde mental e Gênero: relatos de experiência do projeto de extensão Núcleo Maria da Penha-NUMAPE, Irati-PR
}

DOI: $10.5935 / 1984-9044.20200028$

\author{
Katia Alexsandra dos Santos, Monica Karpinski, Izabel Cristina Soares - \\ Universidade Estadual do Centro-Oeste (UNICENTRO)
}

Resumo: A relação entre violência doméstica e saúde mental tem sido apontada em diversos estudos, entre os quais há evidências de que mulheres que passaram por alguma situação de violência têm mais chances de apresentarem sintomas diretamente associados à saúde mental. Por outro lado, problemas relacionados à saúde mental são fatores de risco para a violência doméstica, bem como para a violência institucional, já que há grande probabilidade dessas mulheres não terem suas vozes ouvidas em casos de denúncias.

Considerando essa problemática, este trabalho apresenta relatos de experiência resultantes das pesquisas do projeto de extensão Núcleo Maria da Penha-NUMAPE, discutindo a questão teoricamente e problematizando formas de atendimento e encaminhamento para casos concretos em que estão interseccionadas formas de vulnerabilidade à violência, perpassadas pelas categorias de gênero e também de saúde mental.

PALAVRAS-CHAVE: gênero; saúde mental; violência doméstica; violência contra a mulher; Núcleo Maria da Penha.

\section{Mental Health and Gender: experience reports of the extension project Núcleo Maria da Penha-NUMAPE, Irati-PR}

\begin{abstract}
The relation between domestic violence and mental health has been highlighted in several studies, which show evidences that women who have experienced some situation of violence are more likely to have symptoms directly associated with mental health. On the other hand, problems related to mental health are risk factor for domestic violence, as well as for institutional violence, once there is a high probability of these women not having their voices heard in cases of complaints. Considering this scenario, this work presents an experience report based on the extension project "Núcleo Maria da Penha-NUMAPE", in order to theoretically discuss the issue and problematize ways of care and referral to concrete cases in which the gender violence vulnerability is intersected to mental health.
\end{abstract}

KEY WORDS: gender; mental health; domestic violence; violence against women; Núcleo Maria da Penha 


\section{Introdução}

A

relação entre violência doméstica e saúde mental tem sido apontada em diversos estudos, havendo evidências de que mulheres que passaram por alguma situação de violência têm mais chances de apresentarem sintomas diretamente associados à saúde mental. Entre outras associações possíveis, o estudo de Santos e Monteiro apontou "a propensão ao desenvolvimento de sintomas de pensamentos depressivos aumentou 3,11 vezes com agressão psicológica, 6,13 vezes com agressão física sem sequelas, 2,47 vezes com coerção sexual e 7,3 vezes com agressão física com sequelas" (2018, p. 1).

Quando falamos em saúde mental, é preciso mencionar o período em que predominou o modelo manicomial de tratamento, visto que havia mais mulheres em serviços de atendimento à

\footnotetext{
${ }^{1}$ Projeto de extensão vinculado ao programa
} Universidade Sem Fronteiras (USF/UGF-PR), saúde mental e, ainda assim, não eram considerados os atravessamentos de gênero (Nascimento e Zanello, 2014). Atualmente, é indiscutível a necessidade de pensar saúde mental atrelada a gênero, entre outros marcadores sociais, pelo prisma da interseccionalidade (Crenshaw, 1989; Akotirene, 2019). Contudo, torna-se mais complexo ao pensarmos em atendimentos psicológicos a mulheres em situação de violência, uma vez que questões de saúde mental podem ser fatores que influenciam negativamente no processo de enfrentamento, produzindo efeitos como a deslegitimação de suas falas.

O Núcleo Maria da Penha (NUMAPE) é um projeto de extensão ${ }^{1}$ que oferece atendimento jurídico e psicológico a mulheres em situação de violência doméstica no município de Irati-PR e região. Diante da ausência de uma rede especializada, o NUMAPE busca se 
INTERAÇÕES SOCIAIS DE CRIANÇAS DE TRÊS A QUATRO ANOS EM INSTITUIÇÃO

DE EDUCAÇÃO INFANTIL

articular com as redes municipais de assistência social, educação e saúde, recebendo e realizando encaminhamentos, que podem ser desde atendimento individual até encaminhamentos para grupos de apoio, vinculados à rede de assistência social, por exemplo. Os critérios para atendimento pelo projeto são estar em situação de violência, efetivando ou não a denúncia, e não possuir recursos financeiros para custear despesas de atendimento psicológico ou jurídico.

Durante as supervisões dos atendimentos psicológicos, foi-se percebendo a frequência de casos em que a mulher estava psicologicamente adoecida, o que direcionou a reflexão para as seguintes problemáticas: as experiências cotidianas desiguais entre homens e mulheres geram condições psicológicas distintas; há dificuldade em dimensionar aquilo que é questão de "saúde-doença mental" ${ }^{2}$ (Andrade, 2014) e o que é consequência de uma situação de violência doméstica.

\footnotetext{
${ }^{2}$ Fazemos uso desse termo tal como postulado por Andrade (2014) que, orientando-se em autores como Basaglia (1985) e Amarante (1996), opta por juntar os
}

Ainda que não seja uma tarefa simples, por entrar em campos epistemológicos distintos, é importante distinguir os termos sofrimento psíquico, adoecimento e transtorno mental. A concepção que trazemos neste texto se aproxima do modelo psicossocial de atenção à saúde mental, que compreende o sofrimento mental como condição humana, diferentemente do modelo biomédico. De acordo com Andrade, sofrimento psíquico é considerado como:

não apenas como uma categoria nosográfica, tampouco se resume a um evento biológico ou a um conjunto de sintomas, mas sim como uma experiência subjetiva atravessada pelos modelos e significados do processo de adoecimento e cura atribuídos por cada sujeito e permeada pelas características socioculturais dos contextos em que se desenvolvem (Andrade, 2014, p. 156).

Já adoecimento mental seria um processo em que o sofrimento psíquico vai produzindo efeitos mais duradouros,

termos, colocando-os entre aspas, com o intuito de problematizar a relação saúde-doença. 
INTERAÇÕES SOCIAIS DE CRIANÇAS DE TRÊS A QUATRO ANOS EM INSTITUIÇÃO

DE EDUCAÇÃO INFANTIL

que afetam a vida da pessoa em diferentes esferas, podendo chegar ao que se tem chamado de transtorno mental. Contudo, essas são categorias oriundas dos campos da psicopatologia e da psiquiatria (Dalgalarrondo, 2008), de modo que é preciso colocar sempre em questão essas terminologias.

Mesmo com essa ressalva, entendemos que as questões de saúde-doença mental, quando se aproximam do que é nomeado como transtorno mental, ultrapassam os critérios de atendimento do projeto de extensão por sua complexidade. Essa dificuldade se dá justamente porque "a violência de gênero contra as mulheres não é tratada claramente como um fator de risco para a saúde mental" (Medeiros e Zanello, 2018, s/p), de modo que não se produzem políticas públicas com base em uma perspectiva interseccional.

Tendo em vista essa problemática, este trabalho apresenta relato de experiência a partir de atendimentos realizados no referido projeto de extensão, em que temos discutido teoricamente e procurado estabelecer formas de acompanhamento para casos concretos em que estão interseccionadas formas de vulnerabilidade à violência, perpassadas pelas categorias de gênero e também de saúde-doença mental.

\section{Violência de gênero e saúde mental}

Compreendemos violência de gênero como fenômeno resultante das relações desiguais entre os sexos (Saffioti, 2015), considerando que, aos homens, é destinado o poder e a dominação pela validação de características culturalmente ligadas ao masculino, como a força física e a racionalidade; às mulheres, o oposto: a fragilidade, a sensibilidade e a submissão.

No que tange aos efeitos da violência de gênero à saúde mental das mulheres, é preciso apontar que a discussão de gênero e saúde mental é recente (Nascimento e Zanello, 2014). Acerca disso, produziram-se explicações 
A FONOAUDIOLOGIA ENTRE A OBJETIVIDADE E SUBJETIVIDADE: ATUAÇÃO EM UMA ENFERMARIA DE SAÚDE MENTAL

biologizantes, que remetem ao conceito de histeria (Barbosa, Dimenstein e Leite, 2014), ao invés de se compreender que experiências sociais decorrentes da condição de mulher teriam relação com o adoecimento mental.

A desvinculação da ideia da loucura ao feminino percorre uma longa trajetória: se inicialmente a histeria (derivada da palavra útero) foi compreendida como produto da biologia feminina, posteriormente foi ligada a uma inadequação: ora compreendida como resultante de repressão sexual, ora como masoquismo e até mesmo necessidade de abusos por parte do parceiro (Barbosa, Dimenstein e Leite, 2014). Em contrapartida, os comportamentos masculinos violentos eram pouco considerados nos diagnósticos de sofrimento psíquico das mulheres.

No contexto brasileiro, a compreensão das experiências femininas e, sobretudo, da violência de gênero como fator de risco para o adoecimento mental teve participação fundamental do movimento feminista. À medida que ocorriam as reivindicações e a desconstrução de ideias que concebiam a submissão como intrínseca ao sexo feminino, um novo campo de estudos se desenvolveu, a partir de 1980, resultando em políticas públicas nas áreas da segurança pública, saúde e judiciário, a fim de combater a violência de gênero (Bandeira, 2019).

Atualmente, a política brasileira prevê atendimento integral destinado às mulheres, isto é, uma série de estratégias compostas por políticas e serviços advindos das esferas da saúde, justiça, segurança pública e assistência social. Tais estratégias são nomeadas como rede de enfrentamento e compõem a Política Nacional de Enfrentamento à Violência contra as Mulheres (Brasil, 2011), contudo não há documento que articule especificamente com os serviços de saúde mental. Existe apenas a diretriz de "Encaminhamento para programas de recuperação, para atendimento psicológico e para serviços de saúde mental, quando necessário" (Brasil, 2011, p. 69), que diz respeito ao Serviço de Responsabilização e Educação do Agressor. 

SAÚDE MENTAL

Algumas publicações, entretanto, têm trazido discussões que abordam a questão de gênero, focada explicitamente na mulher, e a de saúde mental. Em pesquisa realizada em um Centro de Atenção Psicossocial (CAPS) de Brasília, Zanello, Fiuza e Costa (2015) constataram que $o$ adoecimento psíquico e o sentido/vivência da doença são distintos entre os usuários e as usuárias entrevistadas: "Enquanto elas se queixam que a doença atrapalha o exercício de cuidado dos filhos e do lar (deveres domésticos), eles sofrem pela não-produtividade" (Zanello, Fiuza e Costa, 2015, p. 240). A pesquisa também evidenciou o relato de sofrimento pelas mulheres, relacionado a diferentes formas de violências, como estupros na infância e/ou adolescência, ou então a ideia de subjugar seu próprio corpo como objeto sexual do homem dentro de relacionamentos. Tais violências podem desencadear sofrimentos relacionados à autoimagem, ao sentimento de culpa, mais ou menos graves, podendo, ainda, produzir o que definimos neste texto como transtorno mental.
A mesma pesquisa de Zanello, Fiuza e Costa (2015) aponta que as mulheres, por buscarem manter o papel feminino do silêncio, da polidez ao falar, acabam por adoecer mentalmente, o que as autoras nomeiam como "implosão". Além disso, o sofrimento causado pelo lugar do silêncio é invisibilizado e desqualificado, afinal não há espaço para dizer o que se sente, muito menos ser agressiva, dentro do paradigma da feminilidade construído em nossa sociedade. Ainda discorrendo acerca das diferenças entre homens e mulheres quando o assunto é saúde mental, CoutoOliveira (2007) afirma, por meio de pesquisa bibliográfica, que "as mulheres são mais afetadas por transtornos de ansiedade, depressão, transtornos obsessivo-compulsivo, transtornos relacionados à somatização, transtornos de pânico e transtornos alimentares" (2007, p. 48). A depressão é alvo de destaque, segundo autores apresentados por Couto-Oliveira (2007), visto que é considerada uma doença predominantemente feminina: “Especialmente entre mulheres pobres, tanto as demandas relacionadas à 
A FONOAUDIOLOGIA ENTRE A OBJETIVIDADE E SUBJETIVIDADE: ATUAÇÃO EM UMA ENFERMARIA DE SAÚDE MENTAL

condição feminina quanto as demandas do contexto de pobreza onde vivem, as tornam mais suscetíveis ao estresse e ao adoecimento físico e mental" (CoutoOliveira, 2007, p. 50). Outros fatores relacionados à condição feminina que afetam a saúde mental das mulheres são "a rigidez da identidade e dos papéis de gênero, a múltipla jornada de trabalho, a discriminação de gênero e a falta de poder em suas relações" (Couto-Oliveira ,2007, p. 50).

Através dessas pesquisas, é possível evidenciar "o quanto as questões geradoras de sofrimento psíquico tem sua base nos estereótipos de gênero" (Zanello, Fiuza e Costa, 2015, p.245). Quando se trata das consequências decorrentes especificamente de violência doméstica contra mulheres, um estudo realizado pela Organização Mundial da Saúde (citado por CoutoOliveira, 2007), "verificou que mulheres que sofrem ou já sofreram violência física e/ou sexual perpetrada por parceiro íntimo, relatam níveis de estresse emocional significativamente maiores que aquelas mulheres não abusadas. Estas mesmas mulheres também estão mais suscetíveis a pensar em suicídio [...]" (Couto-Oliveira, 2007, p. 53).

Estudos como os que trouxemos anteriormente têm sua importância na medida em que materializam a existência de estigmatizações que ainda articulam o lugar da mulher, e ainda mais da mulher pobre, na relação com o patológico. Contudo, é preciso atentar para o perigo de cair em uma espécie de determinismo que reforce esses estereótipos que apontam para a patologização do feminino. Estamos afirmando que a condição de mulher em nossa sociedade é fator de risco para violência, assim como ambos os elementos são fatores de risco para sofrimento mental, o que não quer dizer, todavia, que toda mulher que passa por situação de violência está fadada ao adoecimento psíquico, e que nem toda mulher pobre é vulnerável.

Assim, nossa proposição é levantar debate sobre violência doméstica contra mulheres e saúde-doença mental. Entendemos que é difícil separar aquilo que diz respeito ao adoecimento e/ou transtorno mental - e que precisa ser acompanhado pela rede especializada - 
A FONOAUDIOLOGIA ENTRE A OBJETIVIDADE E SUBJETIVIDADE: ATUAÇÃO EM UMA ENFERMARIA DE SAÚDE MENTAL

daquilo que se compreende como sofrimento mental em consequência da violência doméstica e que, portanto, pode ser atendido pelo Núcleo Maria da
Penha. Esses elementos e distinções tornam complexos nossos critérios para atendimento e encaminhamento.

\section{É caso de violência ou de saúde mental? Como estabelecer critérios para o cuidado integral}

A fim de instrumentalizar nossas práticas, buscamos nos valer de dois elementos teórico-conceituais: o primeiro deles é o conceito de interseccionalidade (Crenshaw, 1989; Akotirene, 2019), a fim de compreendermos que mulher é essa à qual nos referimos e poder deslizar desse aparato conceitual e metodológico para a intersecção de vulnerabilidades, como propomos no título; o segundo é a própria noção de rede, conceito que nos auxilia a pensar no atendimento dessas mulheres que, para ser integral, precisa passar pela rede como um sistema único de cuidado.

O conceito de interseccionalidade, cunhado por Crenshaw (1989) e tomado como instrumento epistemológico pelas feministas negras, "visa dar instrumentalidade teórico-metodológica à inseparabilidade estrutural do racismo, capitalismo e cisheteropatriarcado" (Akotirene, 2019, p. 19). Ou seja, trata-se de um importante instrumento que nos permite vislumbrar categorias fundamentais de marcadores sociais, sem necessariamente separá-las ou hierarquizá-las, mas entendendo que funcionam de forma concomitante, produzindo mais ou menos vulnerabilidade. É por meio desse instrumento que abriga um "sistema de opressão interligado" (Akotirene, 2019, p. 21), portanto, que consideramos ainda um outro marcador social na discussão que ora apresentamos: a questão do 
adoecimento mental. É preciso entender que faz muita diferença quando atendemos uma mulher em situação de violência se ela for uma mulher negra, uma mulher pobre, uma mulher negra e pobre, uma mulher com transtornos mentais e, por fim, uma mulher negra, pobre e com transtornos mentais. Coloca-se aí um conjunto de vulnerabilidades que distancia cada vez mais essa mulher da possibilidade de ser ouvida e ter sua palavra valorizada e/ou minimamente considerada.

É dessa perspectiva que compreendemos que a atenção a esse sujeito também não pode ser feita de maneira fragmentada. Nesse sentido, é fundamental a noção de rede de atendimento, elemento que intersecciona sistemas de atenção organizados pelos equipamentos da assistência social, saúde, segurança pública e judiciário.

A constituição da rede de atendimento e enfrentamento à violência contra a mulher no Brasil, apresentada nas diretrizes gerais (Brasil, 2011), iniciou, efetivamente, apenas em 2003 com a criação da Secretaria de Políticas para Mulheres (SPM), que buscava a garantia de recursos para a criação de serviços e para a implementação de políticas públicas integradas. Após isso foi formulada a Política Nacional de Enfrentamento à Violência contra as Mulheres, que incentivou a formação de redes compostas por todos os serviços que assistem a mulher em situação de violência. Em 2007, o lançamento do Pacto Nacional pelo Enfrentamento à Violência contra as Mulheres consolidou a necessidade de uma rede articulada de atendimento.

Além dessas ações, a promulgação da Lei 11.340/2006 - Lei Maria da Penha - e a criação, em 2005, da Central de Atendimento à Mulher - Ligue 180 também constituíram e fortaleceram a rede de enfrentamento à violência contra mulheres. No 9o artigo da referida Lei fica evidente a necessidade de uma rede estruturada, planejada e intersetorializada:

(...) a assistência à mulher em situação de violência doméstica e familiar será prestada de forma articulada e conforme os princípios e as diretrizes previstos na Lei Orgânica da Assistência Social, no Sistema 
Único de Saúde, no Sistema Único de Segurança Pública, entre outras normas e políticas públicas de proteção (grifo nosso).

A SPM difere dois conceitos quando se trata de rede: a rede de "enfrentamento" à violência e rede de "atendimento" a mulheres em situação de violência. A primeira diz respeito à articulação entre as

(...) instituições/serviços governamentais, nãogovernamentais e a comunidade, visando ao desenvolvimento de estratégias efetivas de prevenção e de políticas que garantam o empoderamento das mulheres e seus direitos humanos, a responsabilização dos agressores e a assistência qualificada às mulheres em situação de violência" (Brasil, 2011, p.7-8).

Já a segunda é definida como o

(...) conjunto de ações e serviços de diferentes setores (em especial, da assistência social, da justiça, da segurança pública e da saúde), que visam à ampliação e à melhoria da qualidade do atendimento; à identificação e ao encaminhamento adequado das mulheres em situação de violência e à integralidade e humanização do atendimento (Brasil, 2011, p. 8).

Desse modo, a rede de enfrentamento, que é composta pela rede de atendimento, é ampla e múltipla devido à complexidade da violência contra as mulheres. A articulação e a integração, através de uma perspectiva intersetorial, é fundamental para que ela seja efetiva e defina os fluxos de atendimentos conforme as especificidades de cada caso e cada região. Cabe destacar, entretanto, que a política nacional em relação às mulheres, como apresentada neste texto, encontra-se em momento de pleno desmonte, devido a elementos de ordem política e ideológica.

A partir dessa explanação acerca da rede de atendimento e enfrentamento à violência contra a mulher, voltamos à pergunta que colocamos neste tópico: os casos atendidos pelo Núcleo Maria da Penha são casos de saúde mental ou casos de atendimento pelos equipamentos ligados às questões de 
violência? "É importante assinalar que poucos campos de conhecimento e atuação na saúde são tão vigorosamente complexos, plurais, interseccionais e com tanta transversalidade de saberes" (Amarante, 2007, p. 14) quanto o campo da saúde mental. Assim, talvez a pergunta não seja exatamente essa, mas: até onde vai a atuação de cada uma das partes? Podemos falar em partes? Como, afinal, atuar de forma integrada?

Além das questões que dizem respeito às especificidades de cada um dos elementos que temos priorizado nesta discussão - o atendimento especializado em situações de violência contra a mulher e o atendimento especializado em saúde mental - outras questões se levantam em função das características locais. Trata-se de um município interiorano, com aproximadamente 60 mil habitantes, o que frequentemente resulta em proximidade informal entre profissionais da psicologia e usuárias. Ocorre, ainda, a necessidade de que haja acompanhamento de familiares próximos na mesma unidade e/ou território de atendimento em saúde mental, o que dificulta, senão impossibilita, os atendimentos psicológicos, uma vez que há apenas três profissionais para abranger o território do município. Isso produz atravessamentos éticos relacionados à profissão, de forma que os critérios fogem a simples elementos técnicos de atuação. Há sempre a possibilidade de encaminhamento para outros órgãos de saúde mental que fazem atendimentos e atividades em grupos, como os Centros de Atenção Psicossocial (CAPS). Contudo, do mesmo modo, faz-se necessária a triagem por meio de atendimento individual.

Desafios também passam pelo fato de que as políticas de atenção às mulheres em situação de violência e as políticas de saúde mental são constituídas e implementadas de maneira separada, de forma que o diálogo necessário para a atenção integral fica precarizado. Assim, quando casos concretos exigem articulação com políticas de saúde, o diálogo é um pouco mais complexo, dentre outros fatores pelo entendimento de que as mulheres assistidas pelo Núcleo já estão sendo atendidas por um ponto da rede e, portanto, não estariam 
em lugar de prioridade no atendimento em saúde mental. Isso se justifica, tendo em vista a carência de profissionais, de modo que o atendimento a todos os casos é praticamente impossível. Assim, ao desligarmos uma usuária, realizando o encaminhamento para a saúde mental do município, sabemos que provavelmente ela será atendida em tempo muito posterior à data do encaminhamento e, ainda, de modo precário.

A fim de exemplificar a problemática, apresentaremos dois casos construídos a partir de experiências de atendimento no Núcleo Maria da Penha. O primeiro tipo de caso diz respeito a usuárias que chegam ao núcleo com uma demanda relacionada à violência doméstica, mas muito rapidamente é possível perceber que esse aspecto não é central nos atendimentos e, sim, questões relacionadas à saúde-doença mental. Como, por exemplo, em situações em que a paciente tem um relato de difícil compreensão devido à desorganização na fala. Nessas situações, muitas vezes a usuária já é encaminhada de outro ponto da rede, o que pode ser entendido como uma dificuldade em ser acolhida e acompanhada devidamente segundo suas necessidades.

Esse é o caso de uma mulher que foi encaminhada informalmente pelo Fórum da Comarca para o Núcleo, já que estava "nervosa" com as questões legais de seu processo (o qual envolvia uma Medida Protetiva, já violada, contra um familiar). Sua queixa inicial era um medo extremo do seu agressor, contudo, já nos primeiros atendimentos, apresentou narrativa desorganizada e, com isso, houve extrema dificuldade por parte da estagiária em compreender qual era a demanda do atendimento. Esses elementos nos deixaram atentas se realmente se tratava de um caso dentro dos critérios do Núcleo.

Diante da situação, nos propusemos a manter $\mathrm{o}$ atendimento psicológico semanal para compreender com maior clareza o caso. Nesse período, a usuária também fora atendida pelo Centro de Referência Especializado de Assistência Social (CREAS), pelo fato de utilizar o dispositivo chamado Botão do Pânico. As profissionais do serviço solicitaram um relatório resumido, a fim de 
compreender o caso, já que houve muita dificuldade para entendê-lo em visita domiciliar. Além desse episódio, vale ressaltar que a usuária fazia uso de psicotrópicos receitados por psiquiatra da rede de saúde, mas não era devidamente acompanhada.

Assim, foi possível perceber ao longo dos atendimentos que o diagnóstico de saúde mental retirou ainda mais o lugar de fala da mulher na rede. Esse caso também é permeado pela pobreza, pois, além do medo de perder a casa para o agressor, a usuária também relatava diversas outras limitações que comprometiam a sua saúde e qualidade mínima de vida, como a impossibilidade de comprar medicamentos, comunicarse e ser compreendida e usar o transporte coletivo. Evidenciou-se, dessa maneira, que os vários pontos que compõem a rede municipal de acolhimento dessa mulher não estavam articulados de maneira efetiva.

Conforme já apontamos anteriormente, a dificuldade em efetivar o encaminhamento para a saúde mental se torna um dos principais entraves em casos em que é inviável a continuidade dos atendimentos pelo Núcleo devido sua complexidade. $\mathrm{O}$ caso foi encaminhado para a saúde mental do município e levou cerca de 3 meses para ser atendido. O que pudemos fazer, no âmbito do projeto de extensão, foi entrar em contato com a usuária $e$ frequentemente contatar o serviço de saúde mental, a fim de garantir o seu atendimento.

O segundo tipo de caso engloba situações em que as usuárias, em decorrência da violência, passam a apresentar sintomas que indicam adoecimento mental. A usuária chegou por demanda de violência sexual, ocorrida há poucos meses. Relatou dificuldades para realizar atividades cotidianas e, principalmente, relacionadas ao sono, como insônias duradouras ou pesadelos. Aos poucos, foi relatando sintomas de ansiedade que se agravaram e evoluíram para crises de pânico. Além disso, apresentou dificuldades de se relacionar socialmente, recusando sair para festas (prática comum para ela anteriormente), especialmente em contextos que envolviam uso de álcool, por relacionar a 
violência que sofrera à própria vulnerabilidade pós consumo de bebidas alcoólicas. Diante dessa problemática, entendeu-se que o acompanhamento psicológico estava no alcance do Núcleo, já que o adoecimento psíquico surgiu, pontualmente, a partir de uma situação de violência, de modo que foi possível trabalhar com estratégias que objetivaram dessensibilizar a usuária da ideia de que estava suscetível à violência o tempo todo e, ao mesmo tempo, desenvolver estratégias de cuidado de si. Nesse caso, encerrada a demanda específica relacionada à situação de violência, foi organizado e discutido com a usuária um plano de encaminhamento para acompanhamento psicoterápico. Assim, o contato com a nova psicóloga foi acompanhado pelo Núcleo. Esse encaminhamento se deu em função de terem surgido outras demandas, além da relacionada à violência, de modo que também fugiu ao domínio de atendimento do projeto, ainda que por motivos diferentes dos abordados no caso anterior.

Por fim, a partir desses casos que ilustram a experiência vivenciada pela equipe da psicologia do Núcleo Maria da Penha, podemos dizer que "a coibição, a prevenção e o atendimento à violência de gênero exigem reflexões e atuações multissetoriais e multidisciplinares, que incidam diretamente na estrutura e na conjuntura do fenômeno organizador da nossa realidade social tão desigual e violenta em relação às mulheres" (Bandeira, 2019, pp. 311-312).

\section{Considerações finais}

Não se trata de definir qual vulnerabilidade é mais presente e, portanto, qual equipamento deverá ser responsável pelo cuidado, mas de compreender que nessa intersecção de vulnerabilidades produz-se a violência. Nessa reflexão, torna-se complexo pensar em causa e efeito, a fim de definir 
o que produz o que: é a violência que produz a condição de sofrimento e/ou adoecimento mental ou é justamente essa condição que coloca a mulher em situação de vulnerabilidade em relação à violência? Cabe olhar caso a caso, não buscando necessariamente uma resposta que identifique causa e efeito, mas procurando estabelecer correlações entre um fenômeno e o outro a fim de dar o devido entendimento da complexidade que entorna a questão da violência de gênero.

Com este texto, tivemos como pretensão materializar, a partir da nossa experiência de atendimento no projeto de extensão Núcleo Maria da Penha NUMAPE, o desafio de falar e atuar de uma perspectiva interseccional em situações de violência doméstica, olhando para casos concretos. Não se trata apenas de encaminhar mulheres com transtorno mental, já que não é simples fazer essa caracterização, do mesmo modo que não é simples definir quando a mulher está ou não está mais em situação de violência. Desse modo, a discussão em torno do conceito de rede que trouxemos neste texto, somada à noção de interseccionalidade que nos ajuda a compreender os diversos elementos que atravessam a constituição dessas mulheres lançam luzes a uma prática que busca se efetivar mediante o compromisso com as usuárias e com o atendimento integral. E isso só pode se dar por meio de articulação efetiva entre os serviços que compõem o cuidado às mulheres em situação de violência, o que é sempre um desafio.

\section{Referências}

Akotirene, C. (2019). Interseccionalidade. São Paulo: Sueli Carneiro; Polén. Coleção Feminismos Plurais, coordenação de Djamila Ribeiro. 
Amarante, P. (2007). Saúde mental e atenção psicossocial. Rio de Janeiro: Editora Fiocruz.

Andrade, A.P.M.D. (2014). "Louca, eu?" : tensionamentos e subversões da/na política pública de saúde mental. In Stevens, C, Oliveira, S.R. D. \& Zanello, V. (2014). Estudos feministas e de gênero: articulações e perspectivas. Ilha de Santa Catarina: Mulheres, pp. 149-160.

Bandeira, L. M. (2019). Violência de gênero: a construção de um campo teórico e de investigação. In Hollanda, H. B. Pensamento feminista brasileiro: formação e contexto (pp. 292-313). Rio de Janeiro: Bazar do Tempo.

Barbosa, L. B., Dimenstein, M. \& Leite, J. F. (2014). Mulheres em situação de violência e seus itinerários em busca de ajuda: um estudo no município de Natal/RN. In Zanello, V. \& Andrade, A. P. M. (Orgs.). Saúde Mental e gênero: diálogos, práticas e interdisciplinaridade. Curitiba: Appris.

Brasil, Secretaria de Política para as Mulheres. (2011). Rede de Enfrentamento à Violência contra as Mulheres. Brasília. Recuperado em:

https://www12.senado.leg.br/institucional/omv/entenda-aviolencia/pdfs/rede-de-enfrentamento-a-violencia-contra-as-mulheres.

Acesso em 30. mar.2020.

Couto-Oliveira, V. (2007). Vida de Mulher. gênero, pobreza, saúde mental e resiliência. (Dissertação de mestrado). Recuperado em:

https://repositorio.unb.br/bitstream/10482/2862/1/22007_VeruscaCoutodeOli veira.pdf

Crenshaw, K. (1989). Demarginalizing the Intersection of Race and Sex. a Black Feminist Critique of Antidiscrimination Doctrine, Feminist Theory and Antiracist Politcs. University of Chicago Legal Forum, n.1, p. 139-167. Recuperado em: https://chicagounbound.uchicago.edu/cgi/viewcontent.cgi?article=1052\&cont ext=uclf. 
Dalgalarrondo, P. (2008). Psicopatologia e Semiologia dos Transtornos Mentais. 2 ed. Porto Alegre: Artmed.

Medeiros, M. P. \& Zanello, V. (2018). Relação entre a violência e a saúde mental das mulheres no Brasil: análise das políticas públicas. Estudose Pesquisas em Psicologia, 18(1). Recuperado em: https://www.epublicacoes.uerj.br/index.php/revispsi/article/view/38128/27578

Nascimento, W. F. \& Zanello, V. (2014). Uma história do silêncio sobre gênero e loucura - parte I: sobre o que não se fala em uma arqueologia do silêncio: as mulheres em História da Loucura. In Zanello, V \& Andrade, A. P. M. (Orgs.). Saúde mental e gênero: diálogos, práticas e interdisciplinaridade. Curitiba: Appris.

Saffioti, H. I. B. (2015). Gênero, Patriarcado e Violência. São Paulo: Editora Fundação Perseu Abramo.

Santos, A. G. \& Monteiro, C. F. S. (2018). Domínios dos transtornos mentais comuns em mulheres que relatam violência por parceiro íntimo. Rev. LatinoAm. Enfermagem. v. 26. Ribeirão Preto. Recuperado em: http://dx.doi.org/10.1590/1518-8345.2740.3099.

Zanello, V., Fiuza, G. \& Costa, H. S. (2015). Saúde mental e gênero: facetas gendradas do sofrimento psíquico. Fractal: Revista de Psicologia, 27(3), 238246. Recuperado em: http://www.scielo.br/pdf/fractal/v27n3/1984-0292fractal-27-3-0238.pdf

Recebido em: 17/08/2020

Aprovado em: 01/12/2020 